\title{
High BMI, Aggressive Tumours and Long Console Time Are Independent Predictive Factors for Symptomatic Lymphocele Formation after Robot-Assisted Radical Prostatectomy and Pelvic Lymph Node Dissection
}

\author{
Christopher Goßler $^{\mathrm{a}}$ Matthias May ${ }^{\mathrm{b}}$ Johannes Breyer ${ }^{\mathrm{a}} \quad$ Gjoko Stojanoski $^{\mathrm{b}}$ \\ Steffen Weikert ${ }^{c}$ Sebastian Lenart ${ }^{d} \quad$ Anton Ponholzer $^{d} \quad$ Christina Dreissig $^{c}$ \\ Maximilian Burger ${ }^{\mathrm{a}} \quad$ Christian Gilfrich $^{\mathrm{b}} \quad$ Johannes Bründl ${ }^{\mathrm{a}}$ \\ Bernd Rosenhammer ${ }^{a}$ \\ aDepartment of Urology, Caritas St. Josef Medical Centre, University of Regensburg, Regensburg, Germany; \\ ${ }^{b}$ Department of Urology, St. Elisabeth Hospital Straubing, Brothers of Mercy Hospital, Straubing, Germany;

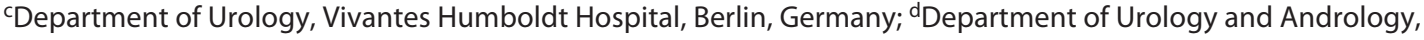 \\ St. John of God Hospital Vienna, Brothers of Mercy Hospital, Vienna, Austria
}

\section{Keywords}

Robot-assisted radical prostatovesiculectomy - Prostate cancer · Lymphocele · Complication

\begin{abstract}
Introduction: Lymphocele (LC) formation is a common complication which may cause severe symptoms after robot-assisted radical prostatovesiculectomy (RARP) with concomitant pelvic lymph node dissection (PLND). Compared to open radical prostatectomy, the amount of data on potential risk factors for LC formation is still limited. The aim of the present study was to identify risk factors for symptomatic LC formation (sLC) after RARP with PLND. Methods: We used the data of a prospective multicentre series of 232 RARP patients which were treated between March 2017 and December 2017. The primary endpoint was the presence of $s L C$ within 90 days. Asymptomatic LC (aLC) formation was also recorded. We evaluated clinical, perioperative, and histopathological criteria and compared their distribution in pa-
\end{abstract}

karger@karger.com www.karger.com/uin

Karger $\stackrel{\text { ! }}{\div}$

GOPEN ACCESS
(C) 2021 The Author(s)

Published by S. Karger AG, Basel

This is an Open Access article licensed under the Creative Commons Attribution-NonCommercial-4.0 International License (CC BY-NC) (http://www.karger.com/Services/OpenAccessLicense), applicable to the online version of the article only. Usage and distribution for commercial purposes requires written permission. tients with and without post-operative sLC. Uni- and multivariable logistic regression analyses (MVAs) were performed to identify potential predictors for LC formation. Regarding the influence of patients' BMI, 2 models were calculated: BMI continuously (model 1 ) and BMI dichotomized with cut-off $30 \mathrm{~kg} / \mathrm{m}^{2}$ (WHO definition, model 2). Results: Post-operative sLC was present in 21 patients $(9.1 \%)$, while aLC was detected in 49 patients (21.1\%) 90 days after RARP with PLND. Patients with $\mathrm{SLC}$ showed higher median baseline PSA levels ( 9.8 vs. $8.1 \mathrm{ng} / \mathrm{mL})$, higher prevalence of obesity (BMI >30; 42.9 vs. $19.9 \%$ ), and longer median console time (180 vs. 165 min) compared to patients without SLC. On MVA higher BMI \{model 1: OR 1.145 (confidence interval [CI] 1.025-1.278); model 2: OR 2.761 (1.045-7.296)\}, longer console time (model 1:OR 1.013 [1.005-1.021]; model 2: OR 1.013 [1.005-1.020]) and an ISUP grade $\geq 3$ (model 1: OR 3.247 [1.182-8.917]; model 2: OR 2.791 [1.050-7.423]) were identified as independent

Johannes Bründl and Bernd Rosenhammer share senior authorship.
Correspondence to:

Christopher Goßler, christopher.gossler@ukr.de 
predictors for SLC development. Conclusion: Patients with aggressive tumours and higher BMI should be informed about a potentially increased risk for $\mathrm{SLC}$ formation. In case of a long console time, a close and regular follow-up should be considered to check for LC development.

(C) 2021 The Author(s)

Published by S. Karger AG, Basel

\section{Introduction}

Prostate cancer represents the second most frequent cancer in men worldwide with 1,276,106 new cases diagnosed in 2018 [1]. Robot-assisted radical prostatovesiculectomy (RARP) has emerged as the new standard of care for the surgical treatment of nonmetastatic prostate cancer. Due to its good oncological and functional results on the one hand and the low perioperative morbidity on the other hand, it has replaced open radical prostatectomy (RPE) in many centres $[2,3]$.

Depending on the patients' preoperative risk profile, intraoperative pelvic lymph node dissection (PLND) is an integral part of the procedure, as it provides the most accurate assessment of metastatic lymph node (LN) involvement. Furthermore, even an oncological benefit has been proposed although conflicting results have been published $[4,5]$. Typically, an extended PLND is performed in patients with high- and intermediate-risk profile as recommended by current guidelines $[6,7]$.

The most common complication after PLND is the formation of lymphoceles (LCs), which are encapsulated collections of lymphatic fluid resulting from the surgical dissection and inadequate closure of lymphatic vessels [4, $5,8]$. LC development has been reported in up to $51 \%$ after RPE with PLND $[4,9]$. Although most LCs remain asymptomatic, systematic screening has been recommended as some may cause relevant problems such as infection, pelvic or leg pain, lower urinary tract symptoms, lower limb oedema, or venous thromboembolism $[4,8,10,11]$. Therapeutic options for patients with clinically symptomatic LC (sLC) include percutaneous drainage (with or without instillation) and open or laparoscopic marsupialization, while asymptomatic patients usually do not need LC treatment.

In previous series, the frequency of sLC formation after RPE with PLND was found to be up to $11.2 \%$ [12-16]. For the robotic approach, somewhat lower rates up to $7.9 \%$ have been reported $[8,9,17,18]$. Several studies aimed to identify potential risk factors for LC formation, but predominantly with regard to open RPE $[12-16,19]$, while only limited data are available for the robotic ap- proach $[8,9,13,20]$. Thus, the purpose of this prospective multicentre RARP series was to further evaluate potential clinical and surgical risk factors for sLC formation.

\section{Patients and Methods}

Study Group and Surgical Technique

The study population consisted of 232 patients who underwent RARP between March 2017 and December 2017 in 1 Austrian and 3 German centres. Data for the current analysis originate from the PIANOFORTE trial (evaluating the impact of a peritoneal flap on LC development after RARP) which has been recently published by our group [21]. The trial has an Ethics Committee's positive vote and was registered in the clinical trials registry (DRKS-ID: DRKS00011115) [22].

All patients presented with clinically organ-confined $\mathrm{PCa}$ and cM0-status. Each RARP was performed in a standardized manner via transperitoneal approach. In all patients, bilateral PLND was performed which included at least the removal of the LNs overlying the external iliac artery and vein as well as the LNs within the obturator fossa cranially and caudally to the obturator nerve. The lymphatic vessels were sealed by bipolar cauterization; clips were placed in addition according to the surgeon's preference. All surgeons had completed their learning curve (>100 RARPs).

\section{Perioperative and Histopathological Aspects}

The following perioperative clinical criteria were recorded and analysed with regard to their effect on post-operative sLC formation: patients' age, Charlson Comorbidity Index (CCI), BMI, baseline PSA levels, history of previous abdominal surgery, console time, in which technique the lymphatic vessels were closed (clips and cauterization vs. cauterization only), and whether or not a peritoneal flap or a nerve-sparing procedure were carried out.

The histopathological samples in the 4 centres were evaluated according to a standardized protocol [23]. For tumour grading, the 2014 ISUP (International Society of Urological Pathology) classification was applied [24]. The histopathological specimens were classified according to the AJC staging system 2017 for PCa (pT and $\mathrm{pN}$ status) [25]. Abdominal drainage, which was placed in all cases, was removed depending on the drainage volume.

The development of sLC within 90 days was diagnosed based on imaging results and patients' symptoms. For LC diagnosis, abdominal/pelvic ultrasound was the primary diagnostic tool, abdominal CT scan was carried out in uncertain cases or when other causes had to be ruled out. A LC was defined symptomatic when either accompanied by signs of infection or by symptoms due to mechanical compression, such as pelvic or leg pain, lower urinary tract symptoms, lower limb oedema, or venous thromboembolism. In all patients, pelvic ultrasound was scheduled at 90 days post-operatively and LC volume was recorded if present. Routine CT scan was not performed in asymptomatic patients.

\section{Statistical Analysis}

Continuous variables were reported as median and interquartile range (IQR), categorical endpoints as absolute and relative frequencies. The Mann-Whitney U test was used to differentiate the distribution of continuous variables between patients with and without 
Table 1. Distribution of various criteria in patients with and without symptomatic lymphocele (within 90 days post-operatively)

\begin{tabular}{|c|c|c|c|c|}
\hline Criteria & $\begin{array}{l}\text { Whole study group } \\
(n=232)\end{array}$ & $\begin{array}{l}\text { Pts. without sLC } \\
(n=211)\end{array}$ & $\begin{array}{l}\text { Pts. with sLC } \\
(n=21)\end{array}$ & $p$ value \\
\hline Median age, years (IQR) & $65(60-70)$ & $66(60-70)$ & $64(58.5-70)$ & 0.623 \\
\hline Median PSA, ng/mL & $8.2(6.0-12.9)$ & $8.1(6.0-12.7)$ & $9.8(7.3-7.7)$ & 0.024 \\
\hline Median BMI, kg/m² (IQR) & $27.2(25.2-29.7)$ & $26.9(25.2-29.4)$ & $29.6(25.6-34.7)$ & 0.053 \\
\hline $\mathrm{BMI} \geq 30 \mathrm{~kg} / \mathrm{m}^{2}, n(\%)$ & $51(22)$ & $42(19.9)$ & $9(42.9)$ & 0.025 \\
\hline Median console time, min (IQR) & $167(129-217)$ & $165(127-215)$ & $180(162.5-281.5)$ & 0.012 \\
\hline Peritoneal flap performed, $n(\%)$ & $108(46.6)$ & $99(46.9)$ & $9(42.9)$ & 0.820 \\
\hline Median number of removed LNs (IQR) & $16(11-21)$ & $15(11-21)$ & $17(11-23)$ & 0.227 \\
\hline \multicolumn{5}{|l|}{ Technique of LND, $n(\%)$} \\
\hline Cauterization only & $83(35.8)$ & $76(36)$ & $7(33.3)$ & \multirow[t]{2}{*}{0.999} \\
\hline Additional clip placement & $149(64.2)$ & $136(64)$ & $14(66.7)$ & \\
\hline \multicolumn{5}{|l|}{ ISUP-GGG, $n(\%)$} \\
\hline $1-2$ & $137(59.1)$ & $128(60.7)$ & $9(42.9)$ & \multirow[t]{2}{*}{0.161} \\
\hline $3-5$ & $95(40.9)$ & $83(39.3)$ & $12(57.1)$ & \\
\hline
\end{tabular}

IQR, interquartile range; LNs, lymph nodes; LND, LN dissection; ISUP-GGG, International Society of Urological Pathology - Gleason grading groups; PSA, prostate-specific antigen; LC, lymphocele; sLC, symptomatic LC; CCI, Charlson Comorbidity Index. Bold values indicate statistical significance.

sLC. The distribution of categorical variables was analysed using the $\chi^{2}$ test (in case of $2 \times 2$ contingency tables: Fisher's exact test).

Uni- and multivariable logistic regression analyses (MVA) were performed on various criteria to evaluate their influence on the endpoints post-operative sLC formation and asymptomatic LC (aLC) formation $>30 \mathrm{~mL}$. Due to the low number of events, the independent effect was analysed by multivariable logistical regression models using a stepwise backward elimination of the independent variables on the basis of the probability of the likelihood-ratio-statistics. The following primary independent variables were selected: age at the time of RARP (continuously in years), CCI ( $\geq 1$ vs. 0), previous abdominal surgery (yes vs. no), console time (continuously in minutes), peritoneal flap performed (yes vs. no), number of LNs removed (continuously), LND technique (clips and cauterization vs. cauterization only), nerve-sparing procedure (yes vs. no), histopathological tumour stage ( $\geq$ pT3 vs. pT2), histopathological LN status ( $\mathrm{pN} 1$ vs. $\mathrm{pN} 0$ ), ISUP Gleason grading group (ISUP-GGG 3-5 vs. 1-2). The BMI was analysed as an independent variable with regard to its effect on LC formation in 2 models: model 1 with the BMI being analysed as a continuous variable and model 2 with a dichotomization in $\geq 30 \mathrm{~kg} / \mathrm{m}^{2}$ versus $<30 \mathrm{~kg} / \mathrm{m}^{2}$ as this cut-off represents the definition of obesity as given by the WHO [26]. For additional analyses of some identified risk factors, enter models were built with covariables of particular interest.

Data analysis was carried out using IBM SPSS Statistics for Windows, version 25.0 (IBM Corp., Armonk, NY, USA). All mentioned $p$ values are 2-tailed; the significance level was defined as $p<0.05$.

Risk Factors for Symptomatic Lymphocele Formation after RARP and PLND

\section{Results}

A total of 232 RARP patients were analysed. Post-operative sLC was present in 21 patients $(9.1 \%)$. Localized pain was observed in 14 cases, infected LC in 9 cases, LC-induced rheological problems in 4 cases, and new-onset LUTS due to the $\mathrm{LC}$ in 1 case. A LC was detected in 49 patients $(21.1 \%)$ 90 days after RARP. The LC volume in those patients was $<30 \mathrm{~mL}$ in 19 patients $(8.2 \%), 31-100 \mathrm{~mL}$ in 17 patients (7.3\%), $101-200 \mathrm{~mL}$ in 8 patients (3.4\%), $201-500 \mathrm{~mL}$ in 2 patients $(0.9 \%)$, and $>500 \mathrm{~mL}$ in 3 patients $(1.3 \%)$.

Table 1 shows a selection of clinical and histopathological study criteria with a subdivision in patients with and without post-operative sLC. The study groups were balanced with regard to age, CCI, and history of previous abdominal surgery. Moreover, no statistically significant differences were observed concerning the median number of removed LNs, LND technique (clip placement vs. cauterization only), pathological tumour stage $>$ pT2, positive nodal status and ISUP-GGG $\geq 3$. The proportion of procedures with nerve-sparing and peritoneal flap was also comparable. In contrast, patients with sLC showed significantly higher baseline PSA levels (median 9.8 vs. $8.1 \mathrm{ng} / \mathrm{mL} ; p=$ 0.024 ), a higher proportion of obese patients ( $42.9 \mathrm{vs} .19 .9 \%$;

Urol Int 2021;105:453-459 
Table 2. Univariable and multivariable analysis of various criteria regarding their influence on the outcome "symptomatic lymphocele within 90 days after RARP”

\begin{tabular}{|c|c|c|}
\hline Criteria & $\begin{array}{l}\text { OR }(95 \% \mathrm{CI}), p \text { value } \\
\text { [univariable] }\end{array}$ & $\begin{array}{l}\text { OR }(95 \% \mathrm{CI}), p \text { value } \\
\text { [multivariable] }\end{array}$ \\
\hline Age (years, continuously) & $0.987(0.928-1.050), 0.681$ & - \\
\hline PSA (ng/mL, continuously) & $1.026(0.991-1.063), 0.147$ & - \\
\hline BMI $\left(\mathrm{kg} / \mathrm{m}^{2}\right.$, continuously $)$ & $1.143(1.029-1.270), 0.013$ & $1.145(1.025-1.278), \mathbf{0 . 0 1 6}[$ model 1$]$ \\
\hline BMI dichotomized ( $\geq 30$ vs. $<30 \mathrm{~kg} / \mathrm{m}^{2}$ ) & 3.018 (1.193-7.633), $\mathbf{0 . 0 2 0}$ & $2.761(1.045-7.296), \mathbf{0 . 0 4 1}[$ model 2] \\
\hline Peritoneal flap performed (yes vs. no) & $1.179(0.477-2.915), 0.722$ & - \\
\hline Number of removed LNs (continuously) & $1.036(0.987-1.088), 0.148$ & - \\
\hline Technique of LND (clips and cauterization vs. cauterization only) & $1.126(0.436-2.911), 0.807$ & - \\
\hline Nerve-sparing procedure (yes vs. no) & $0.650(0.263-1.609), 0.352$ & - \\
\hline Pathological tumour stage (>pT2 vs. pT2) & $1.258(0.484-3.271), 0.637$ & - \\
\hline
\end{tabular}

In model 1 of the multivariable analysis, the BMI is included as a continuous variable, while a dichotomized BMI (cut-off $30 \mathrm{~kg} / \mathrm{m}^{2}$ ) is used in model 2. CI, confidence interval; IQR, interquartile range; LNs, lymph nodes; LND, lymph node dissection; ISUP-GGG, International Society of Urological Pathology - Gleason grading groups; PSA, prostate-specific antigen; RARP, robot-assisted radical prostatovesiculectomy; LC, lymphocele; sLC, symptomatic LC. Bold values indicate statistical significance.

$p=0.025)$ and a longer console time (median 180 vs. 165 $\min ; p=0.012$ ) compared to patients without sLC.

On univariable logistic regression analysis, BMI (continuously, $\left.p=0.013 ; \geq 30 \mathrm{~kg} / \mathrm{m}^{2}, p=0.020\right)$ and console time $(p=0.012)$ were significantly associated with sLC formation. No significant effect was observed for patients' age, CCI, baseline PSA level, previous abdominal surgery, the number of removed LNs, technique of LND, nervesparing or peritoneal flap procedure, pathological tumour stage, pathological LN status, and ISUP-GGG (Table 2).

On MVA (stepwise backwards elimination models), the following 3 parameters remained in the model until the last step and were identified as independent risk factors for sLC formation: patients' BMI (model 1: confidence interval $[\mathrm{CI}] 1.025-1.278, p=0.016$; model 2: CI $1.045-7.296, p=0.041)$, console time (model 1: CI 1.005$1.021, p=0.001$; model 2: CI 1.005-1.020, $p=0.002)$, and ISUP-GGG (model 1: CI 1.182-8.917, $p=0.022$; model 2: CI 1.050-7.423, $p=0.040$ ) (Table 2).

Next, we further investigated the predictive role of console time and ISUP-GGG. We particularly wanted to check if the predictive effect of these variables was potentially driven by a modified surgical LN management. Therefore, again MVAs were performed, but this time we used enter models including the covariables "number of removed LN" and "technique of LND (clips vs. cauteriza- tion only)" into our models. The third included variable was "console time" for the first calculation and "ISUPGGG" (dichotomized) for the second. Console time remained statistically significant $(p=0.002)$ whereas the ISUP-GGG lost its statistical significance $(p=0.180)$. Moreover, the number of removed LNs was examined depending on ISUP-GGG, and here, a median number of 16 LNs (IQR 12-23) in ISUP-GGG 3-5 patients was noted, compared to 15 (IQR 9-20) in ISUP-GGG 1-2. This small difference proved to be statistically significant $(p=0.009)$.

Finally, MVAs (stepwise backwards elimination models) were performed to evaluate potential predictors for aLC development ( $>30 \mathrm{~mL}$ ) at 90 days post-operatively. This cut-off was chosen according to the categories of LC volumes used in the original PIANOFORTE trial and hereby 30 events could be evaluated. Two models were calculated (model 1: BMI continuously; model 2: BMI dichotomized, cut-off $30 \mathrm{~kg} / \mathrm{m}^{2}$ ). In both models, BMI and console time remained in the model until the last step. Higher BMI (model 1: $p=0.002$, OR = 1.157, CI 1.054-1.271; model 2: $p=0.011$, OR 2.910, CI 1.281-6.610) and longer console time (model 1: $p=0.024$, OR $=1.008$, CI 1.001-1.015; model $2: p=0.017, \mathrm{OR}=1.008$, CI 1.001-1.015) turned out to be independent predictive factors for the development of aLC $>30 \mathrm{~mL}$. For all study results, we could prove that centre effects did not impact the findings (data not shown). 


\section{Discussion}

PLND during RPE provides the most accurate assessment of metastatic LN involvement in patients with prostate cancer and may even have a potential oncological benefit although results on this topic were conflicting [4, 5]. The benefits of PLND have to be weighed against a considerable risk of complications. It has been reported that up to $8 \%$ of post-operative complications after RARP with PLND might be directly connected to the PLND [27], with LC formation being the most frequent $[4,5,27]$. Apart from infection, pain, and lower urinary tract symptoms, LCs may cause severe rheological problems. Beyer et al. [11] reported an impaired pelvic vein flow in $21.7 \%$ of their patients with post-operative LC, which proved to be a major risk factor for venous thromboembolism at an odds ratio of almost 3 . In view of these results, they recommended post-operative screening for LCs and therapeutic intervention when LC-related impaired venous flow is observed [11]. Considering the excellent visualization and precise tissue handling, RARP might have the potential to reduce LC rates compared to open RPE although a recent study by Thomas et al. [28] found no differences between the open and the robotic approach.

Several previous studies aimed to identify risk factors for the development of sLC after RPE and PLND, in particular the extent of the PLND, extraperitoneal versus transperitoneal approach, role of metastatic LN involvement, advanced tumour stages, age, anticoagulation management, surgical experience, pelvic drain placement, and closure technique of the lymphatic vessels (clips vs. cauterization only) have been investigated. The majority of these studies, however, focused on open RPE and some aspects still remain inconclusive. Therefore, the purpose of the present study was to provide more data with regard to the robotic approach.

Firstly, we identified a higher BMI as an independent risk factor for aLC and sLC formation in our series. This significant effect was observed applying BMI both as a continuous variable and dichotomized at $30 \mathrm{~kg} / \mathrm{m}^{2}$, which is the WHO definition of obesity. According to our results, obese patients may have an almost 3-fold higher risk for sLC development compared to non-obese patients which is noteworthy for preoperative counselling. This finding is in accordance with a recent study by Sforza et al. [18], which also identified BMI as an independent risk factor for sLC formation following RARP. This may be due to more adipose tissue in the pelvis surrounding the pelvic LNs in obese men. Pre-existing chronic inflammation within this adipose tissue might be triggered by the surgical procedure and con-

Risk Factors for Symptomatic Lymphocele Formation after RARP and PLND tribute to sLC formation [18, 29]. Although some other studies did not show a statistically significant impact $[8,9$, 28 ], the present results strongly underline a considerable influence of the patients' BMI on LC formation.

Secondly, a higher ISUP-GGG significantly influenced sLC development in the present cohort. A possible explanation for this might be the fact that patients with higher ISUP-GGG tend to receive a more extended PLND than patients with lower ISUP-GGG [30]. This hypothesis is underlined by our findings as higher ISUP-GGG lost its statistical significance when the number of removed LNs was included as a covariable on a multivariable logistic regression model. In accordance with that, a slightly higher median number of removed LNs was observed in patients with ISUP-GGG 3-5 compared to ISUP-GGG 1-2 patients. As the extent of the PLND has been shown to considerably influence the risk of LC formation after RPE $[12-14,28]$, we believe that our results concerning the predictive role of more aggressive tumours are at least in part related to a more extensive LN management.

Thirdly, our analyses revealed a longer console time as another independent risk factor for aLC and sLC development. So far, this association has not been reported in literature. As console time kept its statistical significance in the multivariable logistic regression enter model including the number of removed LNs and the PLND technique, it has to be assumed that the console time itself has a considerable impact on LC development. Potentially, such longer procedures may have been caused by adhesions or scarring leading to a more demanding preparation. This might result in increased lymphatic leakage and, therefore, promote LC formation. A validation of this finding in a larger cohort is certainly mandatory.

In contrast to other studies $[12,28]$, we did not find an association of patients' age with sLC development. Moreover, the number of removed LNs did not affect sLC development. Previous studies revealed controversial results regarding this issue. While some studies identified the number of removed LNs as a risk factor for LC formation [12-14, $28]$, other series did not $[8,9,18,20]$. Capitanio et al. [12] reported that the risk of developing a sLC significantly increased when $>20$ LNs were removed. The fact that not all patients in our study received extended PLND (especially in the low-risk group) may explain different findings. In our series, the median number of removed LNs was 16 and only a minority of the patients had $>20$ LNs removed. Nevertheless, the number of removed LNs may become relevant if a more extensive PLND is performed, but this issue remains under debate. Furthermore, the amount of LNs within the pelvic adipose tissue varies within individuals [31], which 
means that the number of LNs removed does not necessarily correlate with the actual extent of the PLND [31].

The role of metastatic LN involvement in LC formation has also been discussed controversially. While some series proposed a relevant influence $[9,20]$, other studies did not and argued that the usually limited nodal involvement in RPE candidates does not cause lymphatic leakage $[12,18$, $19,21]$. In line with the latter, we did not observe any impact of $\mathrm{pN} 1$ status on post-operative LC formation. In our study cohort, however, nodal involvement was present in only 16 patients $(6.9 \%)$, and in view of this limited number, we refrain from drawing definite conclusions.

In terms of lymphatic vessel sealing, Orvieto et al. [9] reported higher LC rates applying only cauterization without any clip placement compared to other series. Therefore, they switched to clips for PLND afterwards [9]. In line with some other series, we found no influence of the sealing technique on LC formation in the present study $[10,16,20]$.

Finally, a surgical modification using a peritoneal flap was suggested to potentially reduce LC formation [32, 33]. The prospective randomized PIANOFORTE trial recently published by our group, however, did not show an influence on post-operative LC rates [21].

The main limitation of the present study is the limited number of patients, which is due to the prospective randomized design of the original PIANOFORTE study. Accordingly, an event size of only 21 patients with sLC $(9.1 \%)$ was present, which had to be taken into account when designing the multivariable logistic regression models. Due to the limited event size, other potential risk factors might not have reached statistical significance in this series as discussed above. Nevertheless, 3 independent predictors for SLC were identified by MVA although only 21 events were present. This certainly underlines the value of these parameters. Certainly, more data on this topic from larger studies are desirable.

\section{Conclusion}

The present study systematically evaluated potential risk factors for LC development and identified 3 independent risk factors for sLC formation after RARP with PLND. Patients with higher ISUP-GGG and higher BMI should be informed about a potentially increased risk for sLC formation during preoperative counselling. In such cases and also in case of a long console time, a close and regular follow-up should be considered to check for LC development and potential complications.

\section{Statement of Ethics}

This study was performed following the approval of the local research Ethics Committee (reference number DRKS00011115).

\section{Conflict of Interest Statement}

The authors declare to have no conflicts of interest.

\section{Funding Sources}

There was no funding for this study.

\section{Author Contributions}

Study concept and design: Bründl, Burger, Gilfrich, and May. Acquisition of data: Stojanoski, Weikert, Lenart, Ponholzer, Dreissig, and Bründl. Analysis and interpretation of data: May, Goßler, and Rosenhammer. Drafting of the manuscript: Goßler and Rosenhammer. Critical revision of the manuscript for important content: May, Bründl, Breyer, and Weikert. Statistical analysis: May, Rosenhammer, and Goßler. Supervision: Bründl and Rosenhammer.

\section{References}

1 Bray F, Ferlay J, Soerjomataram I, Siegel RL, Torre LA, Jemal A. Global cancer statistics 2018: GLOBOCAN estimates of incidence and mortality worldwide for 36 cancers in 185 countries. CA Cancer J Clin. 2018 Nov;68(6): 394-424.

2 Liss MA, Lusch A, Morales B, Beheshti N, Skarecky D, Narula N, et al. Robot-assisted radical prostatectomy: 5-year oncological and biochemical outcomes. J Urol. 2012;188(6): 2205-10.

3 Dell'Oglio P, Mottrie A, Mazzone E. Robotassisted radical prostatectomy vs. open radical prostatectomy: latest evidences on perioperative, functional and oncological outcomes. Curr Opin Urol. 2020;30(1):738.

4 Lee HJ, Kane CJ. How to minimize lymphoceles and treat clinically symptomatic lymphoceles after radical prostatectomy. Curr Urol Rep. 2014 Oct;15(10):445.

5 Fossati N, Willemse PM, Van den Broeck T, van den Bergh RCN, Yuan CY, Briers E, et al. The benefits and harms of different extents of lymph node dissection during radical prostatectomy for prostate cancer: a systematic review. Eur Urol. 2017;72(1):84-109.

6 Mottet N, Bellmunt J, Bolla M, Briers E, Cumberbatch MG, De Santis M, et al. EAU-ESTRO-SIOG guidelines on prostate cancer.
Part 1: screening, diagnosis, and local treatment with curative intent. Eur Urol. 2017 Apr;71(4):618-29.

7 Sanda MG, Cadeddu JA, Kirkby E, Chen RC, Crispino T, Fontanarosa J, et al. Clinically localized prostate cancer: AUA/ASTRO/SUO guideline. Part II: recommended approaches and details of specific care options. J Urol. 2018;199(4):990-7.

8 Keskin MS, Argun ÖB, Öbek C, Tufek I, Tuna MB, Mourmouris $\mathrm{P}$, et al. The incidence and sequela of lymphocele formation after robot-assisted extended pelvic lymph node dissection. BJU Int. 2016 Jul;118(1): 127-31. 
9 Orvieto MA, Coelho RF, Chauhan S, Palmer KJ, Rocco B, Patel VR. Incidence of lymphoceles after robot-assisted pelvic lymph node dissection. BJU Int. 2011 Oct;108(7):1185-90.

10 Liss MA, Skarecky D, Morales B, Osann K, Eichel L, Ahlering TE. Preventing perioperative complications of robotic-assisted radical prostatectomy. Urology. 2013;81(2):319-23.

11 Beyer J, Wessela S, Hakenberg OW, Kuhlisch E, Halbritter K, Froehner M, et al. Incidence, risk profile and morphological pattern of venous thromboembolism after prostate cancer surgery. J Thromb Haemost. 2009 Apr;7(4): 597-604.

12 Capitanio U, Pellucchi F, Gallina A, Briganti A, Suardi N, Salonia A, et al. How can we predict lymphorrhoea and clinically significant lymphocoeles after radical prostatectomy and pelvic lymphadenectomy? Clinical implications. BJU Int. 2011;107(7):1095-101.

13 Naselli A, Andreatta R, Introini C, Fontana V, Puppo P. Predictors of symptomatic lymphocele after lymph node excision and radical prostatectomy. Urology. 2010;75(3):630-5.

14 Musch M, Klevecka V, Roggenbuck U, Kroepfl D. Complications of pelvic lymphadenectomy in 1,380 patients undergoing radical retropubic prostatectomy between 1993 and 2006. J Urol. 2008;179(3):923-9.

15 Gotto GT, Yunis LH, Guillonneau B, Touijer K, Eastham JA, Scardino PT, et al. Predictors of symptomatic lymphocele after radical prostatectomy and bilateral pelvic lymph node dissection. Int J Urol. 2011;18(4):291-6.

16 Heers H, Laumeier T, Olbert PJ, Hofmann R, Hegele A. Lymphoceles post-radical retropubic prostatectomy: a retrospective evaluation of epidemiology, risk factors and outcome. Urol Int. 2015;95(4):400-5.

17 Horovitz D, Lu X, Feng C, Messing EM, Joseph JV. Rate of symptomatic lymphocele formation after extraperitoneal vs. transperitoneal robot-assisted radical prostatectomy and bilateral pelvic lymphadenectomy. J Endourol. 2017 Oct;31(10):1037-43.
18 Sforza S, Tellini R, Grosso AA, Zaccaro C, Viola L, Di Maida F, et al. Can we predict the development of symptomatic lymphocele following robot-assisted radical prostatectomy and lymph node dissection? Results from a tertiary referral centre. Scand J Urol. 2020 Jun 26;54(4):328-33.

19 Khoder WY, Trottmann M, Buchner A, Stuber A, Hoffmann S, Stief CG, et al. Risk factors for pelvic lymphoceles post-radical prostatectomy. Int J Urol. 2011;18(9):638-43.

20 Lee JY, Diaz RR, Cho KS, Yu HS, Chung JS, Ham WS, et al. Lymphocele after extraperitoneal robot-assisted radical prostatectomy: a propensity score-matching study. Int J Urol. 2013;20(12):1169-76.

21 Bründl J, Lenart S, Stojanoski G, Gilfrich C, Rosenhammer B, Stolzlechner M, et al. Peritoneal flap in robot-assisted radical prostatectomy: results of a multicenter, randomized, single-blind study (PIANOFORTE) of the efficacy in reducing postoperative lymphocele. Dtsch Arztebl Int. 2020;117(14):243-50.

22 Available from: https://www.drks.de/drks_ web/navigate.do? navigationId=trial.HTML\& TRIAL_ID=DRKS00011115. Accessed 2020 Jul 22.

23 Van der Kwast TH, Amin MB, Billis A, Epstein JI, Griffiths D, Humphrey PA, et al. International Society of urological pathology (ISUP) consensus conference on handling and staging of radical prostatectomy specimens. Working group 2: T2 substaging and prostate cancer volume. Mod Pathol. 2011;24(1):16-25.

24 Epstein JI, Egevad L, Amin MB, Delahunt B, Srigley JR, Humphrey PA. The 2014 International Society of urological pathology (ISUP) consensus conference on gleason grading of prostatic carcinoma: definition of grading patterns and proposal for a new grading system. Am J Surg Pathol. 2016; 40(2):244-52.

25 Amin MB, Greene FL, Edge SB, Compton CC, Gershenwald JE, Brookland RK, et al. The eighth edition AJC cancer staging manual: continuing to build a bridge from a population-based to a more "personalized" approach to cancer staging. CA Cancer J Clin. 2017; 67(2):93-9.
26 World Health Organization. Fact sheet: obesity and overweight. Retrieved from: https:// www.who.int/news-room/fact-sheets/detail/ obesity-and-overweight. Accessed 2020 Aug 17.

27 Ploussard G, Briganti A, de la Taille A, Haese A, Heidenreich A, Menon M, et al. Pelvic lymph node dissection during robotassisted radical prostatectomy: efficacy, limitations, and complications-a systematic review of the literature. Eur Urol. 2014;65(1): $7-16$.

28 Thomas C, Ziewers S, Thomas A, Dotzauer R, Bartsch G, Haferkamp A, et al. Development of symptomatic lymphoceles after radical prostatectomy and pelvic lymph node dissection is independent of surgical approach: a single-center analysis. Int Urol Nephrol. 2019;51(4):633-40.

29 Nishimura S, Manabe I, Nagai R. Adipose tissue inflammation in obesity and metabolic syndrome. Discov Med. 2009;8(41):55-60.

30 Liss MA, Palazzi K, Stroup SP, Jabaji R, Raheem OA, Kane CJ. Outcomes and complications of pelvic lymph node dissection during robotic-assisted radical prostatectomy. World J Urol. 2013;31(3):481-8.

31 Weingärtner K, Ramaswamy A, Bittinger A, Gerharz EW, Vöge D, Riedmiller H. Anatomical basis for pelvic lymphadenectomy in prostate cancer: results of an autopsy study and implications for the clinic. J Urol. 1996; 156(6):1969-71.

32 Lebeis C, Canes D, Sorcini A, Moinzadeh A. Novel technique prevents lymphoceles after transperitoneal robotic-assisted pelvic lymph node dissection: peritoneal flap interposition. Urology. 2015;85(6):1505-9.

33 Stolzenburg JU, Arthanareeswaran VKA, Dietel A, Franz T, Liatsikos E, Kyriazis I, et al. Four-point peritoneal flap fixation in preventing lymphocele formation following radical prostatectomy. Eur Urol Oncol. 2018; 1(5):443-8.
Risk Factors for Symptomatic Lymphocele Formation after RARP and PLND
Urol Int 2021;105:453-459 